\title{
Detection of Gene Mutation for Patients with McCune- Albright Syndrome and Diagnosis Experience: A Report of Two Cases and Literature Review
}

\author{
Sun $\mathrm{L}^{1 *}$, Yan $\mathrm{S}^{2}$, Gao $\mathrm{H}^{1 *}$, Yuan $\mathrm{C}^{1,2}$, WangJ ${ }^{3}$, \\ Zhang J ${ }^{1}$ and ${\mathrm{Wu} \mathrm{D}^{4}}^{4}$ \\ ${ }^{1}$ Department of Pediatrics and Adolescent Gynecology, \\ The Children's Hospital, Zhejiang University School of \\ Medicine, National Clinical Research Center for Child \\ Health, Hangzhou, China \\ ${ }^{2}$ School of Public Health, Zhejiang University School of \\ Medicine, Hangzhou, China \\ ${ }^{3}$ Oncology Surgery Department, The Children's Hospital, \\ Zhejiang University School of Medicine, National Clinical \\ Research Center for Child Health, Hangzhou, China \\ ${ }^{4}$ Genetics \& Metabolism Department, The Children's \\ Hospital, Zhejiang University School of Medicine, \\ National Clinical Research Center for Child Health, \\ Hangzhou, China \\ \#Contributed Equally to this work \\ *Correspondling author: Liying Sun, Department of \\ Pediatrics and Adolescent Gynecology, The Children's \\ Hospital, Zhejiang University School of Medicine, \\ National Clinical Research Center for Child Health, \\ Binsheng Road 3333, Hangzhou, Zhejiang, China
}

Received: August 03, 2021; Accepted: August 19, 2021; Published: August 26, 2021

\begin{abstract}
Background: McCune-Albright Syndrome (MAS) is a rare disease characterized by a broad spectrum of syndromes, including hyperfunctioning endocrinopathies, café-au-lait leisions, and multiple bone fibrous dysplasias. The diagnosis of MAS is usually based on clinical symptoms. However, most of MAS patients may not display typical symptoms, which make the early diagnosis and treatment of MAS difficult.
\end{abstract}

Cases: Patient 1, a 7-year and 4-month-old girl, developed vaginal bleeding with no apparent cause. A Café-au-lait macule patch could be seen on the right trunk. E2 was 914pmol/L. FSH $<0.1 / \mathrm{U} / \mathrm{L}$, Luteinizing Hormone $(\mathrm{LH})<0.1 \mathrm{IU} / \mathrm{L}$. Performing ultrasound, there was a cystic mass in the left accessory area with ovarian echo. There were bone abnormalities in the long bones of the extremities. Patient 2, a girl aged 4 years and 9 months, has abnormal enlarged breasts with no obvious cause. There was a cystic mass in the left accessory area with ovarian echo No apparent Café-au-lait macule hyperpigmented skin macule was seen. E2 is $800 \mathrm{pg} / \mathrm{ml}, \mathrm{FSH}<0.1 \mathrm{IU} / \mathrm{L}$, LH is $0.2 \mathrm{IU} / \mathrm{L}$. GNAS mutation were both positive in the cyst tissue but was negative in the peripheral blood.

Conclusion: Gene sequencing is the confirmable proof in diagnosis of MAS. Negative GNAS gene mutation results in the peripheral blood and skin cannot completely rule out the possibility of MAS. Biopsy of the lesion tissues, such as ovarian tissue, may present more efficient results. New detection techniques have higher sensitivity and make molecular diagnosis more accurate.

Keywords: Gene mutation; McCune-Albright syndrome; Fibrous dysplasia

\section{Background}

Fibrous dysplasia/McCune-Albright syndrome (FD/MAS) is rare, heterogeneous, clinical disease. Three typical features of MAS are hyperpigmented skin macules, hyperfunctioning endocrinopathies and Polyostotic Fibrous Dysplasia (PFD). Other also associated with abnormalities in the cardiovascular system, liver and gallbladder, and kidneys [1]. The diagnosis of MAS can be made based on clinical features. However, the broad spectrum of clinical presentations makes the diagnosis difficult, which brings a great challenge for clinicians. When MAS fails to be confirmed clinically, a molecular diagnosis of affected tissues is indicated [2]. But detecting rate is influenced by the sample origins and the sensitivity of the detection technique [3].

\section{Case Presentation}

Patient 1, a seven years and four months old girl, developed vaginal bleeding with no apparent cause half a month ago. The amount of vaginal bleeding was a little, lasting for three days, with no obvious discomfort such as hematuria, bloody stools, and abdominal pain. Physical examination: height $124.5 \mathrm{~cm}$, weighed $26.8 \mathrm{~kg}$, Tanner II breasts with palpable $3-4 \mathrm{~cm}$ small indurations. Public hair was in Tanner Stage I, with estrogenizing of the hymen and white vaginal discharge. A Café-au-lait macule can be seen on the right trunk. On March 23, 2020, ultrasound of the uterus and ovary was performed, which showed that the size of the uterus was $3.9^{\star} 2.6^{\star} 2.3 \mathrm{~cm}$, the cervix was $2.8 \mathrm{~cm}$, and the thickness of the double-layer endometrium was $0.83 \mathrm{~cm}$. The right ovary measured $2.3^{*} 1.1^{\star} 1.0 \mathrm{~cm}$. The left accessory area had a cystic mass of $4.0^{*} 3.4^{*} 2.3 \mathrm{~cm}$, with sound penetration and a peripheral size of $1.8^{\star} 0.6 \mathrm{~cm}$ ovarian echo. An anteroposterior cranial radiograph and an extremity long bone radiograph were performed. Small nodular high-density shadows were seen in the soft tissue overlap area of the right medial femur. There were obvious bone abnormalities in the long bones of the extremities, and no obvious abnormalities were seen in the front and lateral positions of the skull. Pelvic MRI showed a cyst in the left adnexal area, which was probably from the ovary. The uterus was enlarged. E2 increased significantly, which was $914 \mathrm{pmol} / \mathrm{L}$. FSH $<0.1 \mathrm{IU} / \mathrm{L}$, Luteinizing Hormone $(\mathrm{LH})<0.1 \mathrm{IU} / \mathrm{L}$. Tumor markers were negative. After obtaining the informed consent of the parents of the patient $1 \mathrm{ml}, 3 \mathrm{ml}$ of peripheral blood (EDTA anticoagulation) of the patient was taken. At the same time, laparoscopic surgery was performed to obtain a biopsy of the cyst wall. The proteinase $\mathrm{K}$ method was used to extract genomic DNA from peripheral blood and cyst wall tissues of children. Sequencing results were analyzed using SeqMan software for sequence comparison. The gene sequencing result of the first patient showed that the GNAS gene was c.601C > T (p.R201C) with a low-grade variant (C:T=227:28) in the cyst tissue. However, the gene mutation was not detected in the peripheral blood.
Austin J Obstet Gynecol - Volume 8 Issue 7 - 2021

Submit your Manuscript | www.austinpublishinggroup.com

Sun et al. (C) All rights are reserved
Citation: Sun L, Yan S, Gao H, Yuan C, Wang J, Zhang J, et al. Detection of Gene Mutation for Patients with McCune-Albright Syndrome and Diagnosis Experience: A Report of Two Cases and Literature Review. Austin J Obstet Gynecol. 2021; 8(7): 1190. 
Patient 2, a girl aged 4 years and 9 months was found breast development with no obvious cause 3 years ago, with no obvious tenderness, no history of eating "honey or royal jelly" and other supplements, no history of obvious growth acceleration, no history of exposure to contraceptives. Physical examination: height $102.6 \mathrm{~cm}$, weight $16.2 \mathrm{~kg}$, Tanner II breasts with hard nodules with a diameter of about $2 \mathrm{~cm}$. The vulva was in the process of development, the hymen ring was thickened and edema. No obvious Café-au-lait macule was seen. Auxiliary examination: E2 is $800 \mathrm{pg} / \mathrm{ml}, \mathrm{FSH}<0.1 \mathrm{IU} / \mathrm{L}, \mathrm{LH}$ is $0.2 \mathrm{IU} / \mathrm{L}$. Breast B ultrasound: right breast measured $2.8^{\star} 2.2^{\star} 1.0 \mathrm{~cm}$, left breast measured $2.8^{\star} 2.6^{\star} 1.0 \mathrm{~cm}$. Uterus ovarian ultrasound: the size of the uterus was $2.7^{\star} 2.5^{\star} 1.1 \mathrm{~cm}$, cervix was $1.8 \mathrm{~cm}$, the endometrial membrane was invisible. The right ovary was $1.7^{\star} 0.7^{\star} 0.6 \mathrm{~cm}$; the left ovary was $2.8^{\star} 2.1^{\star} 2.7 \mathrm{~cm}$, with a $2.8^{\star} 1.3^{\star} 1.4 \mathrm{~cm}$ cystic mass with sound penetration. Bone age: backward. This case was considered peripheral precocious puberty. Follow-up for 3-6 months, we found both breasts and vulvar were in development status, with left ovarian cysts. Left ovarian cyst and sex hormone levels changed irregularly. The gene sequencing result of this patient showed that the GNAS gene was c.602G >A (p.R201H) with a low-grade variant $(\mathrm{G}: \mathrm{A}=13: 190)$ in the cyst tissue. However, the gene mutation was not detected in the peripheral blood.

\section{Discussion}

The genetic mechanism of the MAS is related to somatic activating mutations of the GNAS gene, which encodes the guanine nucleotide binding protein (G protein) alpha subunit (Gsa) [4]. The mosaic nature of MAS results in variability in the clinical presentation. If mutations happen early during embryogenesis stage, the influenced tissue is widespread [3]. Typical MAS patients presents with a triad of physical signs: autonomous hyperfunction of the endocrine system, café-au-lait lesions and PFD of the bone.

Currently, the diagnosis of MAS is based on characteristic features: presenting with 2 or more clinical features [2]. However, if mutations occur late during embryogenesis, the tissue involved is limited and the patients may not present with classic characteristics [3]. In a study of 113 MAS patients, only $24 \%$ presenting the classic triad, and $33 \%$ with two symptoms, $40 \%$ with only one symptom [5] The symptoms of MAS are heterogeneous and atypical MAS accounts for a large proportion. The diagnosis is challenging. If clinical, radiological and histological analysis fails to confirm the diagnosis of MAS, a molecular diagnosis of affected tissues can be performed [2]. The detection rate of mutations depends on the mosaic pattern in the tissue and the sensitivity of the technique.

The detection rate of peripheral blood and skin was low. Due to the convenience of sampling, serum leukocytes are generally used for molecule detection. However, because of low level of mosaicism, genetic testing for peripheral blood is not sensitive and always leads to false-negative results [6,7]. In the study, when peripheral blood was tested, positive rates were $46 \%, 21 \%$ and $8 \%$ for patients with classic triad, two signs, and only one sign respectively. Although one patient showed all the typical symptoms of MAS, the result of the genetic analysis in peripheral leucocytes was negative [8]. The detection rate of damaged skin was also not high, only $27 \%$ (3 of 11) skin samples were positive [5]. And even in the patients with three typical symptoms, the detection rate was only $14.3 \%$ ( 1 of 7 patients) [5].
If invasive sampling is available to the patient or his/her family, biopsy can be performed on the lesion tissue, which will present higher positive rate. In a study in 2004 , almost $90 \%$ of patients presenting the classic triad showed positive results, and the figures were $94 \%$ and $89 \%$ in patients with two and one sign, respectively. Among them, $77 \%$ of ovaries, $68 \%$ of ovarian cyst fluid, $80 \%$ of bone tissue, and $50 \%$ of adrenal glands can be tested as positive [5]. Pienkowski once reported a case of a 3-year-old girl with endocrine abnormality. However, the X-ray of her bones was normal and there was no Café-au-lait macule. Percutaneous puncture of simple ovarian cyst, the result of genetic analysis was positive [9]. In our first case, the results in the peripheral blood was negative, but the results of sample on the cyst wall obtained a positive result.

The molecular diagnosis of MAS is also relative to the sensitivity of the detection technique. By sanger sequencing, mutation cannot be detected in leukocytes [7]. Pyrosequencing is a simple and accurate quantification method, the detection sensitivity of which can reach $95 \%$ [10]. Next generation sequencing allows researchers to analyze genome from small amount of DNA. A study found that mutation detection rate of the Peptide Nucleic Acid (PNA), Next Generation Sequencing (NGS) and combinatory use of PNA and NGS (PNANGS) was $56 \%, 63 \%$ and $75 \%$, respectively. Thus, the PNA-NGS method may be effective to detect low-abundance GNAS mutation [11]. 3'-modified oligonucleotides - polymerase chain reaction and combining Real-Time COLD- and MAMA-PCR TaqMan techniques are also feasible and efficient molecular techniques $[12,13]$. The PNAclamping method and the Peptide Nucleic Acid (PNA) probe with fluorescent labeling can improve the sensitivity of current assays $[3,14]$. The digital PCR ( $\mathrm{dPCR})$ can increase the detection rate of gene mutation alterations, especially in the blood [15]. The use of highly sensitive droplet digital PCR (ddPCR) is breakthrough [7]. When detecting the peripheral blood, the positive rate of ddPCR technology can reach $77.42 \%$, which is higher than real-time fluorescent pyrophosphorolysis-Activated Polymerization technology (PAP) (29.03\%) and second-generation sequencing (56.25\%) [16].

\section{Conclusion}

MAS is an extremely heterogeneous disease, genetic testing can be used as a valuable adjunct to help diagnose disease. The positive results depend on the origin of sample and the sensitivity of different molecular detection technologies. Negative GNAS gene mutation results from the sample of the peripheral blood and skin cannot completely rule out the possibility of MAS. Biopsy of the lesion tissues may present more effective clinical results. New technologies like ddPCR bring more hope for molecular detection.

\section{References}

1. Boyce AM and MT Collins. Fibrous Dysplasia/McCune-Albright Syndrome: A Rare, Mosaic Disease of Gas Activation. Endocr Rev. 2020; 41: 345-370.

2. Javaid MK, et al. Best practice management guidelines for fibrous dysplasia/ McCune-Albright syndrome: a consensus statement from the FD/MAS international consortium. Orphanet J Rare Dis. 2019; 14: 139.

3. Hou JW. McCune-Albright Syndrome: Diagnosis and clinical course in eleven patients. Pediatr Neonatol. 2018; 59: 418-420.

4. Weinstein LS, et al. Activating mutations of the stimulatory $G$ protein in the McCune-Albright syndrome. N Engl J Med. 1991; 325: 1688-1695.

5. Lumbroso S, F Paris and C Sultan. Activating Gsalpha mutations: analysis 
of 113 patients with signs of McCune-Albright syndrome--a European Collaborative Study. J Clin Endocrinol Metab. 2004; 89: 2107-2113.

6. Tufano M, et al. Auxological and Endocrinological Features in Children with McCune Albright Syndrome: A Review. Front Endocrinol (Lausanne). 2020; 11: 522 .

7. Romanet $P$, et al. Using Digital Droplet Polymerase Chain Reaction to Detec the Mosaic GNAS Mutations in Whole Blood DNA or Circulating Cell-Free DNA in Fibrous Dysplasia and McCune-Albright Syndrome. J Pediatr. 2019 205: 281-285.e4

8. Medina YN and R Rapaport. Evolving diagnosis of McCune-Albright syndrome. atypical presentation and follow up. J Pediatr Endocrinol Metab. 2009; 22: 373-377

9. Pienkowski C, et al. Recurrent ovarian cyst and mutation of the $\mathrm{Gs}$ alpha gene in ovarian cyst fluid cells: what is the link with McCune-Albright syndrome? Acta Paediatr. 1997; 86: 1019-1021.

10. Liang Q, et al. Quantitative analysis of activating alpha subunit of the protein $(\mathrm{Gs \alpha})$ mutation by pyrosequencing in fibrous dysplasia and other bone lesions. J Mol Diagn. 2011; 13: 137-142.
11. Narumi S, et al. Quantitative and sensitive detection of GNAS mutations causing mccune-albright syndrome with next generation sequencing. PLoS One. 2013; 8: e60525.

12. Cho EK, et al. Clinical and endocrine characteristics and genetic analysis of Korean children with McCune-Albright syndrome: a retrospective cohort study. Orphanet J Rare Dis. 2016; 11: 113.

13. de Sanctis L, et al. Combining Real-Time COLD- and MAMA-PCR TaqMan Techniques to Detect and Quantify R201 GNAS Mutations in the McCuneAlbright Syndrome. Horm Res Paediatr. 2017; 87: 342-349.

14. Lo FS, TL Chen and CC Chiou. Detection of Rare Somatic GNAS Mutation in McCune-Albright Syndrome Using a Novel Peptide Nucleic Acid Probe in a Single Tube. Molecules. 2017; 22: 1874.

15. Elli FM, et al. Improved Molecular Diagnosis of McCune-Albright Syndrome and Bone Fibrous Dysplasia by Digital PCR. Front Genet. 2019; 10: 862.

16. Wenyi Xie, et al. Application of digital droplet PCR in detection of activating hotspot mutations in GNAS gene in children with McCune-Albright syndrome. Chinese Journal of Endocrinology and Metabolism. 2020; 36: 416-420. 